\title{
Large Area Perovskite Light-Emitting Diodes by Gas-Assisted Crystallization
}

Vittal Prakasam, ${ }^{\text {ac }}$ Daniel Tordera, ${ }^{\text {a }}$ Francesco Di Giacomo, ${ }^{\text {b }}$ Robert Abbel, ${ }^{a}$ Arjan Langen, ${ }^{a}$ Gerwin Gelinck, ad and Henk J. Bolink*c

Halide perovskites have been gaining considerable attention recently for use in light-emitting applications, due to their bandgap tunability, color purity and low cost fabrication methods. However, current fabrication techniques limit the processing to small-area devices. Here, we show that a facile $\mathrm{N}_{2}$ gas-quenching technique can be used to make methylammonium lead bromide-based perovskite light-emitting diodes (PeLEDs) with a peak luminance of $6600 \mathrm{~cd} / \mathrm{m}^{2}$ and a current efficiency of $7.0 \mathrm{~cd} / \mathrm{A}$. We use this strategy to upscale PeLEDs to large-area substrates $\left(230 \mathrm{~cm}^{2}\right)$ by developing a protocol for slot-die coating combined with gas-quenching. The resulting large area devices (9 devices of each $4.46 \mathrm{~cm}^{2}$ per substrate) with three slot-die coated layers exhibit uniform emission with a peak luminance of $550 \mathrm{~cd} / \mathrm{m}^{2}$ and a current efficiency of $2.6 \mathrm{~cd} / \mathrm{A}$. The reasons for the reduced performance and improvement routes are discussed. These results mark a vital step towards scalable manufacturing techniques for PeLEDs

a. Holst Centre, High Tech Campus 31, 5656 AE, Eindhoven, The Netherlands.

b. TNO, partner in Solliance, High Tech Campus 21, 5656 AE, Eindhoven, The Netherlands.

c. Instituto de Ciencia Molecular, Universidad de Valencia, C/Catedrático J. Beltrán 2, 46980 Paterna, Spain.

d. Department of Applied Physics, Eindhoven University of Technology, 5600 MB Eindhoven, The Netherlands.

+ Electronic Supplementary Information (ESI) available: Materials; Perovskite synthesis; Experimental section; Device stack; Layer Thickness; Optical characterization; AFM; SEM; XRD; J$V$-L of devices and statistics. See DOI: 10.1039/x0xx00000x

\section{Introduction}

Since the first demonstration of electroluminescence at room temperature from organicinorganic metal halide perovskites1,2 (referred to as "perovskites"), this class of materials has attracted considerable attention for light-emitting applications3-10. Among the beneficial functional properties of perovskites are the bandgap tunability11,12, the balanced charge carrier mobilities13 and color-pure emission with a full-width at half maximum (FWHM) of less than $20 \mathrm{~nm} 14$. Perovskite light emitting diodes (PeLEDs) have been demonstrated with tuneable emission across the visible spectrum11,15,16 by tailoring the emitting perovskite layer composition17-20 and dimensionality (for example, addition of ammonium-halide ligands) $4,7,10,21,22$. External quantum efficiencies (EQE) of over $20 \%$ have been obtained, placing these devices on par with organic and quantum dot light-emitting diodes23. Furthermore, similar materials with lower bandgaps have been deposited from low cost precursor solution by large-area techniques such as slot-die coating and integrated into solar cells24,25.

So far, most of the PeLED fabrication methods involve the use of spin-coating technique and, frequently, the use of a sacrificial anti-solvent (also called as "nano-crystal pinning") step26, both of which are not readily scalable to large substrate sizes. Consequently, alternative techniques must be explored. Recently, a film formation technique has been demonstrated 
where rapid crystallization is induced by forcing an N2 flow towards the substrate, referred to as gas-assisted crystallization or gas-quenching (GQ)27,28. Slot-die coating along with gasquenching has been used successfully to make perovskite photovoltaic (PV) devices with $12 \%$ power conversion efficiency (PCE) over $10 \mathrm{~mm} 2$ of device area29. This makes it timely to look into the strategies for upscaling PeLED fabrication to large areas, preferably through costeffective solution processable coating techniques.

In this report, we develop the gas-quenching technique for PeLEDs and demonstrate the fabrication of large area PeLEDs (devices of $4.46 \mathrm{~cm} 2$ over a fully-coated substrate of $230 \mathrm{~cm} 2$ ) using slot-die coating in combination with gas-quenching. The influence of different key processing parameters on the perovskite film structure and properties are thoroughly studied by electrical and morphological characterization. We demonstrate PeLEDs based on the archetype 3D perovskite, methylammonium lead bromide (MAPbBr3) fabricated by gasquenching of a wet film of precursor solution in dimethyl sulfoxide (DMSO). The gas pressure and the delay time between precursor film deposition and the start of the gas-quenching play a crucial role in the final perovskite film morphology and device performance. In particular, smooth and uniform films of MAPbBr3 comprising of small crystallites $(60 \mathrm{~nm})$ are obtained by initiating the gas quenching process as soon as possible after the coating has been completed, at a gas inlet pressure of 6 bar. Using these optimized conditions, spin-coated PeLEDs with an active area of $16 \mathrm{~mm} 2$ were fabricated, showing a peak luminance of $6600 \mathrm{~cd} / \mathrm{m} 2$ and current efficiencies of $7.0 \mathrm{~cd} / \mathrm{A}$.

Finally, the compatibility of this approach with large area processing methods was demonstrated by the fabrication of PeLEDs (with an active area of $4.46 \mathrm{~cm} 2$ ) using slot-die coating on a substrate as large as $230 \mathrm{~cm} 2$. These PeLEDs show peak luminances of $550 \mathrm{~cd} / \mathrm{m} 2$ and current efficiencies of $2.6 \mathrm{~cd} / \mathrm{A}$.

\section{Experimental}

\section{Device Fabrication}

The PeLED device stack and the corresponding energy levels of the different materials are illustrated in Fig. $1 \mathrm{~b}$ and S1 (ESI + ). PeLEDs were devised with four functional layers sandwiched between an indium tin oxide (ITO) anode and a LiF/Al cathode. A Poly(3,4ethylenedioxythiophene)-poly(styrenesulfonate) (PEDOT:PSS) layer was used as the hole injection layer (HIL). In order to confine the charges effectively, a $30 \mathrm{~nm}$ layer of N4,N4'(Biphenyl-4,4'-diyl)bis(N4'-(naphthalen-1-yl)-N4,N4'- diphenylbiphenyl-4,4'-diamine) (Di-NPB) was used as the hole transport layer (HTL), and a $30 \mathrm{~nm}$ evaporated layer of 1,3-bis[3,5di(pyridin-3-yl)phenyl]benzene (BmPyPhB) as electron transport layer (ETL). The energy levels of these wide-bandgap organic transport layers were well suited to contain the injected charges within the MAPbBr3 layer. The choice and thickness of the different layers of the device were optimized in our previous work20. The MAPbBr3 films were either spin-coated (small area devices) or slot-die coated (large-area devices) on top of the HTL from a DMSO solution using the gas-quenching method as illustrated in Fig. 1a. We used a non-stoichiometric precursor, with an excess of methylammonium bromide $(\mathrm{MABr})$ to lead bromide $(\mathrm{PbBr} 2)$ in a 2:1 ratio. An excess of $\mathrm{MABr}$ has been proven to aid in the formation of small crystallites and enhances device performance for PeLEDs3,20. After deposition, all perovskite films were annealed for 2 mins at $80^{\circ} \mathrm{C}$. 


\section{Characterization}

All device characterizations were conducted in a glovebox under N2 environment. The current density-voltage (J-V) characteristics were measured using a Keithley 2400 source meter. Simultaneously, the luminance was measured using a calibrated silicon photodiode (against a Minolta LS-110 luminance meter) connected to a Keithley 6485 Picoammeter, at a step rate of $200 \mathrm{mV}$. The electroluminescence spectra were obtained using an Oceanoptics USB 2000+ spectrophotometer. For morphological characterization, the $\mathrm{MAPbBr} 3$ films were spin-coated using the gas-quenching method on a $30 \mathrm{~nm}$ layer of Di-NPB to replicate the device morphology. For atomic force microscopy (AFM), the samples were loaded into a Dimension Fast Scan (Bruker) AFM sample hood which was maintained at a relative humidity level of $<0.1 \%$ by continuously flushing with $\mathrm{N} 2$ gas. The scans were performed, using a Si probe in tapping mode ( $\mathrm{k} 4 \mathrm{~N} / \mathrm{m}$, frequency $350 \mathrm{kHz}$ ). X-ray diffraction (XRD) patterns from $10^{\circ}-70^{\circ}$ were obtained using a Panalytical $X^{\prime}$ Pert MPD pro diffractometer at a scan rate of $2^{\circ}$ min-1. Scanning electron microscope (SEM) images were captured in a Zeiss Supra 55VP equipment using the in-lens detector. More details can be found in the experimental section of the electronic supplementary information (ESI $)$.

a)

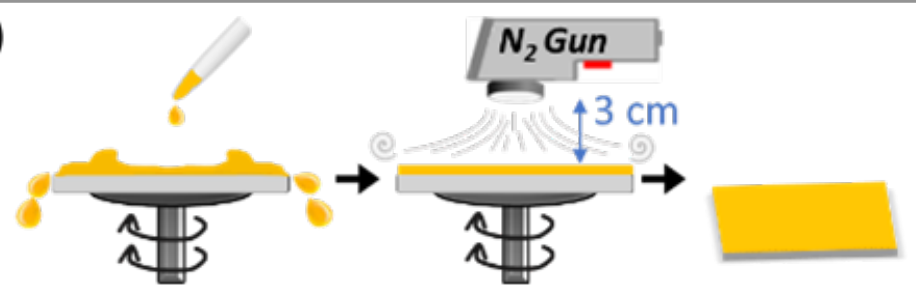

b)

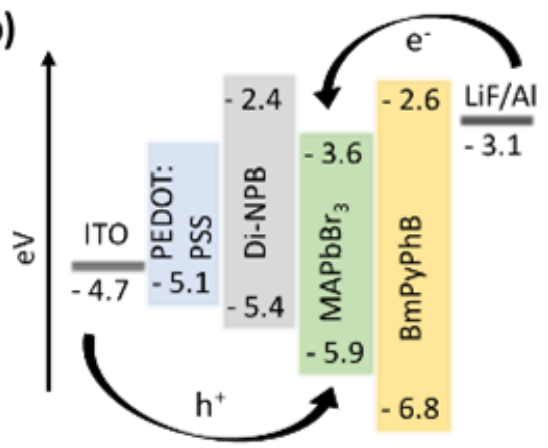

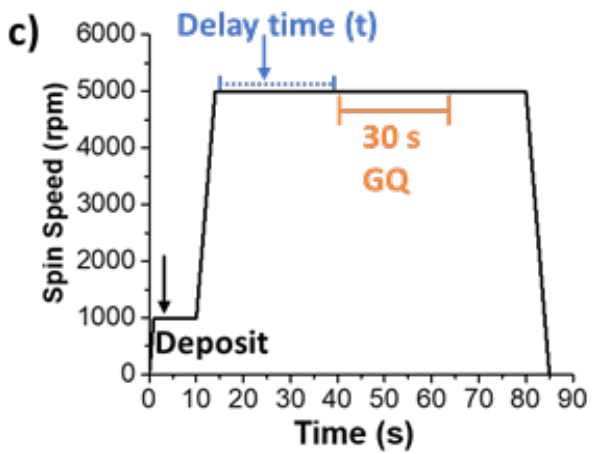

Fig. 1 (a) Schematic illustration of the processing steps using spin-coating and gas-quenching technique. (b) Energy levels of the different layers of the PeLEDs reported in this work. (c) Representative spin curve with process steps, and their timings, use used for depositing the perovskite film.

\section{Results and Discussion}

The optimal crystal structure and morphology required for an efficient PeLED emission depends on the perovskite film processing parameters. Hence, spin-coating was used on small-sized substrates to explore the effect of key gas-quenching processing parameters on the perovskite films' physical and optical properties. ITO/glass substrates were prepared with PEDOT:PSS/DiNPB bilayer coatings to mimic the morphology of films in final devices. Perovskite precursor solution was spin-coated on top of the Di-NPB layer and subsequently gas-quenched. A typical 
spin curve used to deposit the perovskite layer by gas-quenching is shown in Fig. 1c. The precursor solution is dispensed on the substrate spinning at $1000 \mathrm{rpm}$ at two seconds into the process (indicated by black arrow). Then, the rotation speed is ramped up to $5000 \mathrm{rpm}$ in order to form a well-defined wet film. At this stage, a variable delay time $(t)$ is introduced ranging from $0 \mathrm{~s}$ to $35 \mathrm{~s}$, during which the film is left spinning undisturbed at $5000 \mathrm{rpm}$. After the delay time has elapsed, a nitrogen gun, held at $3 \mathrm{~cm}$ above the spinning substrate, directs a flow of N2 gas towards the perovskite film for a fixed duration of $30 \mathrm{~s}$. The N2 gas inlet pressure was set at 2, 4 or 6 bar. The N2 flow causes the remaining solvent to swiftly evaporate, forcing the solution to supersaturate and the perovskite crystallites to precipitate. A high rate of supersaturation leads to the formation of a large number of homogenous nuclei resulting in smooth and uniform films $27,28,30$. One way to control the rate of supersaturation is by tuning the delay time during the spin-coating process. We decided to systematically study the effect of gas-quenching delay timings from $0 \mathrm{~s}$ to $35 \mathrm{~s}$, in steps of $5 \mathrm{~s}$.

Fig. S2 (ESI + ) shows the thicknesses of the resulting perovskite films deposited at various gas pressures and delay timings. The thickness increases from $\sim 120 \mathrm{~nm}$ to $\sim 180 \mathrm{~nm}$ with increasing delays from $0 \mathrm{~s}$ to $35 \mathrm{~s}$, respectively. No significant influence of gas-pressure was observed on the film thicknesses under otherwise identical conditions. The resulting films exhibit an absorption band edge at around $\sim 540 \mathrm{~nm}$ (Fig. S3, ESI + ). The characteristic excitonic feature in the absorption spectra is visible at $\sim 520 \mathrm{~nm}$ for the 6 and 4 bar pressure whereas it is irresolvable for the 2 bar gas pressure. Therefore, in the remainder of the study we focused mainly on the maximum $\mathrm{N} 2$ pressure of 6 bar. The photoluminescence $(\mathrm{PL})$ spectra show peaks centred around $\sim 530 \mathrm{~nm}$ (Fig. S3, ESI+), indicative of the formation of a MAPbBr3 perovskite. These results illustrate that the band gap of the films are largely unaffected by the change in gas-pressure or delay time.

To gain more insight into the microstructure, we investigated the surface morphology and topography of the AFM and SEM, as shown in Fig. 2a and 2b for the films fabricated with 6 bar gas pressure. The AFM images reveal clear morphological differences between the different samples. Up to a delay time of $20 \mathrm{~s}$ there is a gradual increase in the crystallite size, and the surface roughness increases from $4.6 \mathrm{~nm}$ to $11 \mathrm{~nm}$ (Fig. 2c). For longer delay times (>20 s), the crystallites tend to agglomerate into $\mu \mathrm{m}$ sized grains and the surface roughness increases drastically from $\sim 12 \mathrm{~nm}$ to $\sim 44 \mathrm{~nm}$. SEM imaging further confirm that the apparent $\mu \mathrm{m}$ sized grains in the AFM images are actually composed of smaller crystallites that are agglomerated. These trends are observed for all the gas pressures (see ESIt Fig. S4 for 2 bar and 4 bar). For short delay timings $(<20 \mathrm{~s})$, the surface roughness of the perovskite films are comparable to values reported for anti-solvent based methods 4,20 . We propose that gas-quenching induces a high level of supersaturation for $\mathrm{t}<20 \mathrm{~s}$ and after ca. $20 \mathrm{~s}$, the rate of heterogeneous nucleation is faster than the supersaturation. For the PeLEDs fabricated with the conventional anti-solvent process, the anti-solvent dripping timing lies beyond $30 \mathrm{~s}$ into the spinning process to achieve the best results, even for similar precursors $3,4,20$. Hence, these findings suggest that the dynamics of perovskite film formation depend on the specific crystallization technique employed. 


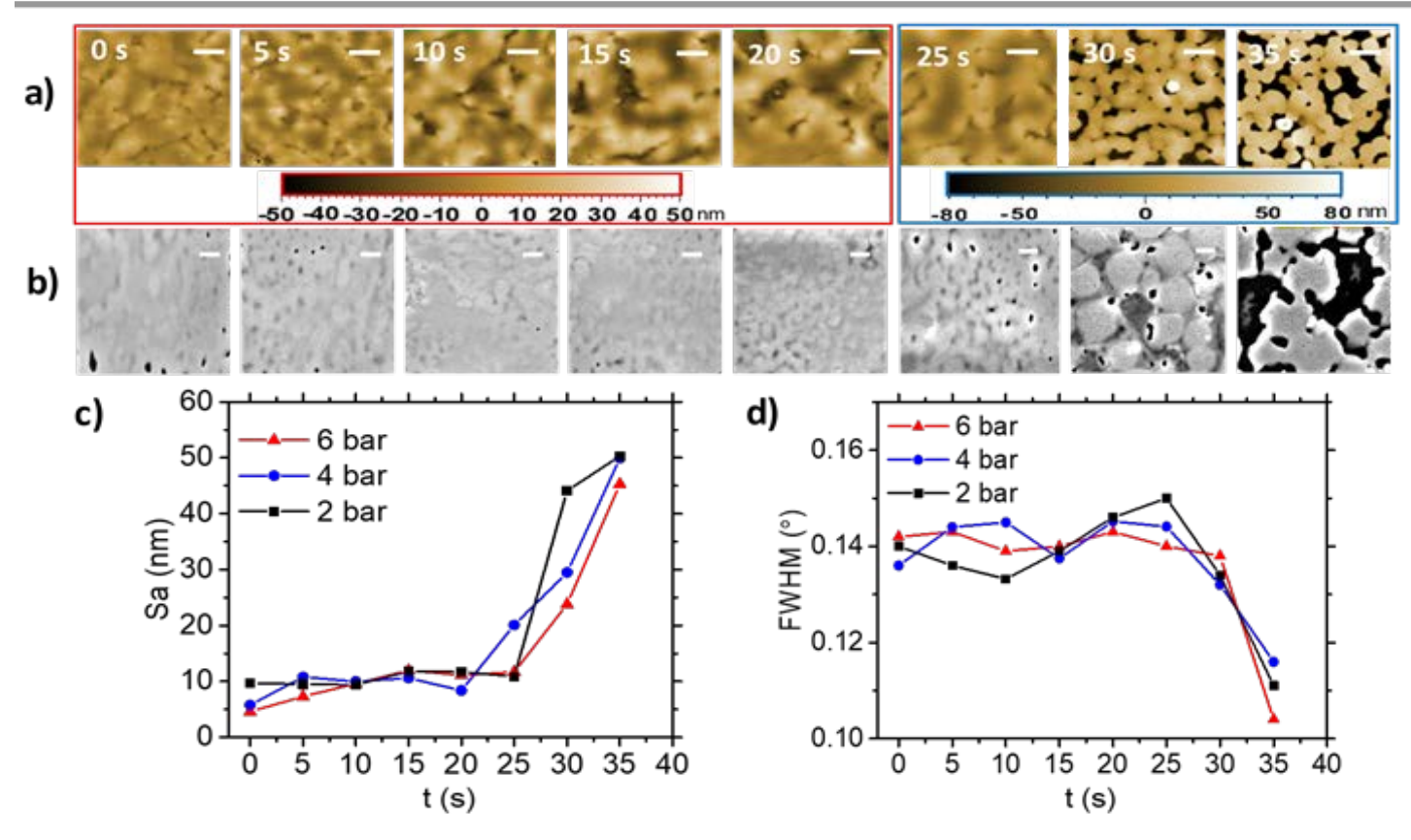

Fig. 2 (a) AFM images of the MAPbBr3 films prepared by gas-quenching at a pressure of 6 bar and various delay timings, marked on the top left corner. The scale bars in the images represent $2 \mu \mathrm{m}$. The $z$-axis roughness scale is provided at the bottom of the images grouped together by the colored box (red or blue). The scan area of the images is $10 \times 10 \mu \mathrm{m} 2$. (b) SEM images of the corresponding films as indicated in the AFM images. The scale bars represent $400 \mathrm{~nm}$. (c) The mean area roughness as a function of delay timings for various gas pressures, obtained from an AFM scan over an area of $10 \mu \mathrm{m} 2$. (d) FWHM of the (100) XRD peaks at $15^{\circ}$ vs. delay timings for various gas pressures.

XRD measurements were performed to determine the structure and crystallinity of the perovskite films coated on a PEDOT:PSS/Di-NPB bilayer. The films exhibit peaks at $15.1^{\circ}, 30.3^{\circ}$ and $46.1^{\circ}$ (Fig. S5, ESIt) that are typically attributed to the (100), (200) and (300) planes; respectively, of a stable Pm5 $\mathrm{m}$ cubic phase31. No trace of the precursor materials was detected indicating a complete conversion of the precursors into perovskite films. The position of the XRD peaks are independent of $\mathrm{GQ}$ delay timings and gas pressures, within the ranges studied, confirming that the bulk properties of the material are unchanged. The FWHM of the $15.1^{\circ}$ peaks shows no significant change with respect to delay timings between $0 \mathrm{~s}$ to $25 \mathrm{~s}$ for the different gas pressures, as shown in Fig. 1d, i.e., the crystallite sizes within these films are rather similar. From the FWHM of the (100) peak, we estimate the average crystallite size of the perovskite films made with delay times between $0 \mathrm{~s}$ to $25 \mathrm{~s}$ to be $\sim 60 \mathrm{~nm}$ using the Scherrer equation. Beyond delay time of $25 \mathrm{~s}$, however, the FWHM gradually decreases indicating a definite increase in the crystallite size, in line with our SEM findings. These results indicate that to achieve smooth perovskite films with uniform crystallites gas-quenching needs to be initiated as soon as possible after the wet layer deposition.

Next, spin-coated MAPbBr3 PeLEDs with an active area of $16 \mathrm{~mm} 2$ were prepared using the gasquenching technique. As an initial test, the $\mathrm{MABr}$ to $\mathrm{PbBr} 2$ ratio was varied between 1:1, 1.5:1, 2:1, 2.5:1 and 3:1. This was done to check if our rationale behind using excess $\mathrm{MABr}$ holds true for gas-quenching technique20. The PeLEDs were prepared using a gas pressure of 6 bar and with a delay time of $15 \mathrm{~s}$. Increasing the $\mathrm{MABr}: \mathrm{PbBr} 2$ ratio from 1:1 to 2:1 resulted in a ten-fold enhancement of maximum luminance from $245 \mathrm{~cd} / \mathrm{m} 2$ to $2680 \mathrm{~cd} / \mathrm{m} 2$, respectively (Fig. S6, 
ESIt). These results are in agreement with what has been reported for antisolvent quenching20. We thus continued to prepare spin-coated PeLEDs with MABr:PbBr2 ratio of 2:1 at various gas pressures and delay timings as described for the initial tests.
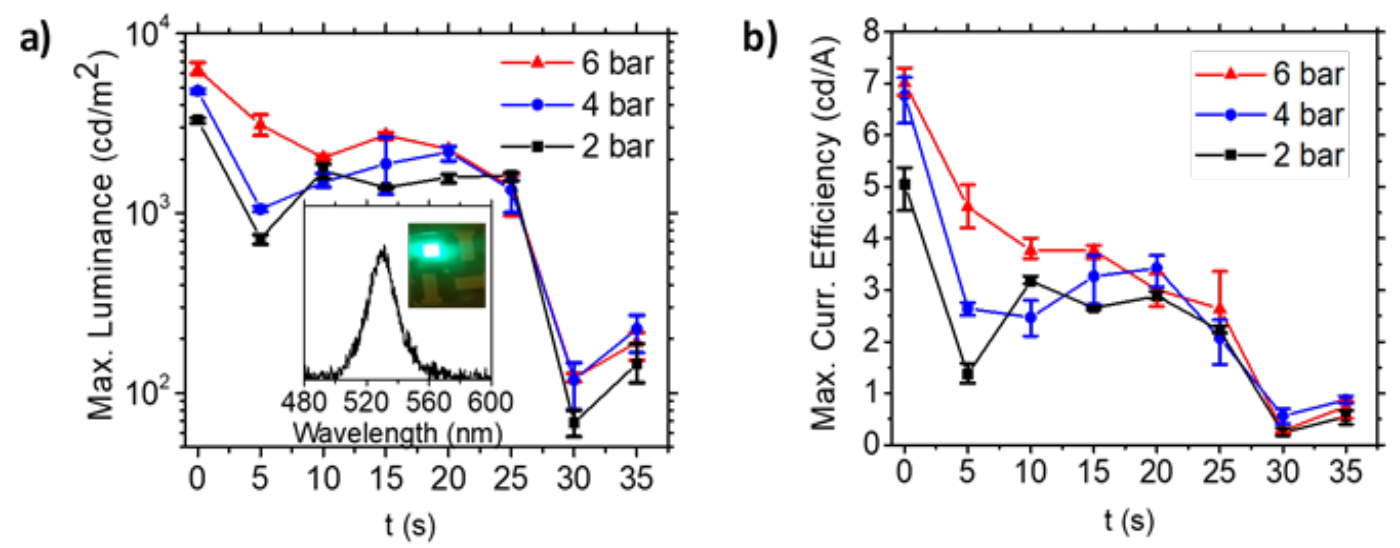

Fig. 3 (a) Average maximum luminance and (b) average maximum current efficiency of the PeLEDs vs. delay timings, measured for different gas pressures. Inset: The electroluminescence spectra of a typical device under $5 \mathrm{~V}$ bias exhibiting green emission.

The completed PeLEDs have turn-on voltages between 3 and $3.4 \mathrm{~V}$ and exhibit low leakage currents of $<2.7 \times 10-4 \mathrm{~mA} / \mathrm{cm} 2$ as shown in the current density-voltage-luminance (J-V-L) curves in Fig. S7 (ESI + ). They exhibit characteristic green electroluminescence centered around $532 \mathrm{~nm}$ with a FWHM of $\sim 20 \mathrm{~nm}$ (inset Fig. 3). Fig. 3a and 3b show the maximum luminance and maximum current efficiencies of the PeLEDs, at different gas pressures and gas-quenching timings. Each data point represents an average value of three devices fabricated in different batches. The PeLEDs fabricated with delay time between $0 \mathrm{~s}$ to $20 \mathrm{~s}$ show better performance in terms of luminance and current efficiencies than those with delay times longer than $25 \mathrm{~s}$. Immediate gas quenching $(\mathrm{t}=0 \mathrm{~s})$ resulted in high-performance devices with peak luminance and current efficiencies of $6600 \mathrm{~cd} / \mathrm{m} 2$ and $7.0 \mathrm{~cd} / \mathrm{A}$, respectively. Smaller crystallites are generally thought to be favorable as they result in better spatial charge confinement and enhanced bi-molecular radiative recombination3,20. This explains the enhanced performance of these devices having small crystallites in line with the AFM, SEM and XRD analysis. Beyond 25 $s$ the performance drops to undesirable values, irrespective of the gas pressures. This can be explained by the fact that beyond $25 \mathrm{~s}$ the films are rather non-uniform and discontinuous, as discussed earlier. We also note that the gas pressure has a minor effect on the device performance within the range studied, with a slight preference for higher gas pressure. We propose that a high gas-pressure (6 bar) leads to rapid evaporation of the solvent and will accelerate the rate of supersaturation resulting in uniform crystallite growth. It is also noteworthy that the trends in maximum luminance for various gas pressures largely agrees with the trend observed in the FWHM of the XRD peaks (Fig. 2d), confirming the higher performance of PeLED when smaller crystals are used. Hence, this corroborates our earlier conclusions derived from the XRD analysis on the films. Our findings indicate that in order to prepare PeLEDs using the gas-quenching technique, the quenching step must be carried out as soon as possible, and at a high gas-pressure. 
As a next step, we decided to scale up the process to large areas using the slot-die coating technique. It offers efficient material usage, high throughput, compatibility with roll-to-roll production and low production cost32. Slot-die coating has been used previously to make efficient organic light emitting diodes 33-35 and perovskite solar modules with an active area of $12.5 \times 13.5 \mathrm{~cm} 2$ and PCE above $10 \% 36$. Slot-die coating combined with gas-quenching was used to fabricate perovskite solar cells with PCE of $11.96 \% 29$. We use this technique to deposit $\mathrm{MAPbBr} 3$ layers on $15.2 \times 15.2 \mathrm{~cm} 2$ substrates containing 9 devices of each $4.46 \mathrm{~cm} 2$ active area. A schematic of the coating process is shown in Fig. 4a. The precursor solution is pumped through a slot-die head to form a meniscus with the substrate. By moving the slot-die over the substrate (indicated with a red arrow), a uniform, continuous wet layer is deposited. The thickness of the wet layer depends on the ink flow rate and the speed of the slot-die movement with respect to the substrate. The substrates with a patterned ITO anode layer were first slotdie coated with a PEDOT:PSS layer of $100 \mathrm{~nm}$ and subsequently a Di-NPB layer of $60 \mathrm{~nm}$. The thicknesses were deliberately chosen to be thicker than the spin-coated small devices to avoid side-leakage current and to reduce shunt paths in case of any particle contamination. Then, a $0.27 \mathrm{M} \mathrm{MAPbBr} 3$ precursor solution from DMSO (MABr:PbBr2 - 2:1) was slot-die coated on top of the Di-NPB layer inside a nitrogen filled glove box at room temperature. The top-surface of the Di-NPB layer was treated with a N2 plasma to ensure good wettability of the precursor solution. Directly after the perovskite coating a N2 knife was used to start the gas-quenching process at 6 bar pressure. The N2 knife was moved back and forth manually over the entire coated area at a speed of $\sim 300 \mathrm{~mm} / \mathrm{s}$ for $60 \mathrm{~s}$ at a distance of ca. $3 \mathrm{~cm}$ from the substrate. This resulted in a $200 \mathrm{~nm}$ thick dry MAPbBr3 film. As with the other layers, the perovskite layer thickness was targeted to be higher than in the small area spin-coated devices $(80 \mathrm{~nm})$. The XRD patterns of the slot-die coated film (Fig. 4c) exhibits peaks at $15.0^{\circ}, 21.5^{\circ}, 30.2^{\circ}, 34^{\circ}, 37.3^{\circ}, 43.4^{\circ}$ and $46^{\circ}$ that are typically assigned to (100), (110), (200), (210), (211), (220) and (300) planes, respectively, indicating a cubic $\mathrm{Pm} 3 \mathrm{~m}$ phase 3 , similar to the spin coated films. From the $15.0^{\circ}$ peak, using the Scherrer equation we estimate the crystallite size to be $\sim 68 \mathrm{~nm}$ which is slightly higher than the crystallite size of the spin coated devices $(60 \mathrm{~nm})$. Subsequently, the devices were completed by depositing the BmPyPhB (ETL) and the LiF/Al cathode layers by thermal evaporation, similar to the small area PeLEDs. 

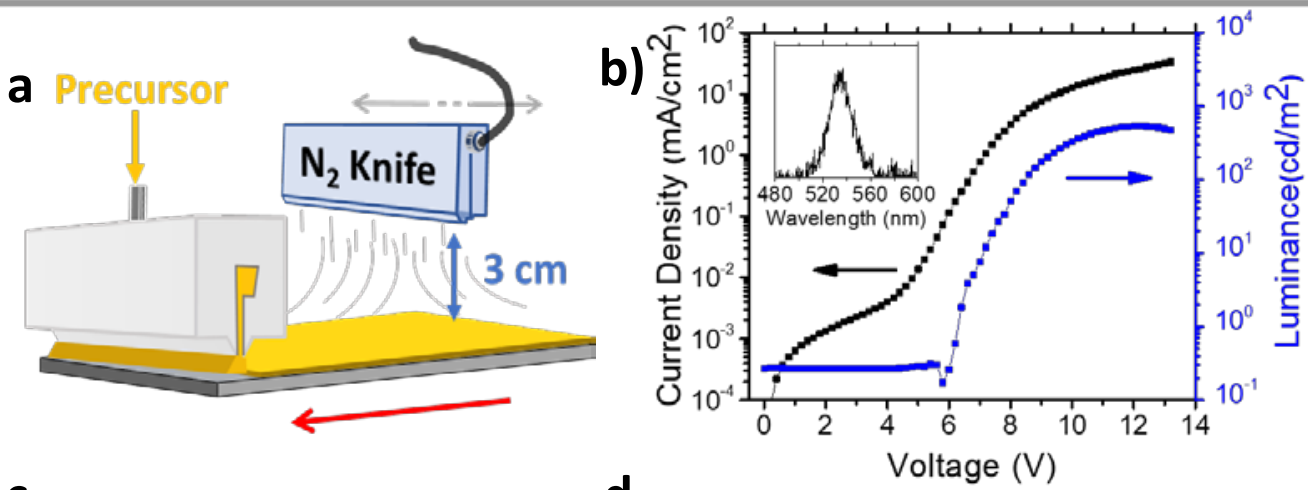

C

d
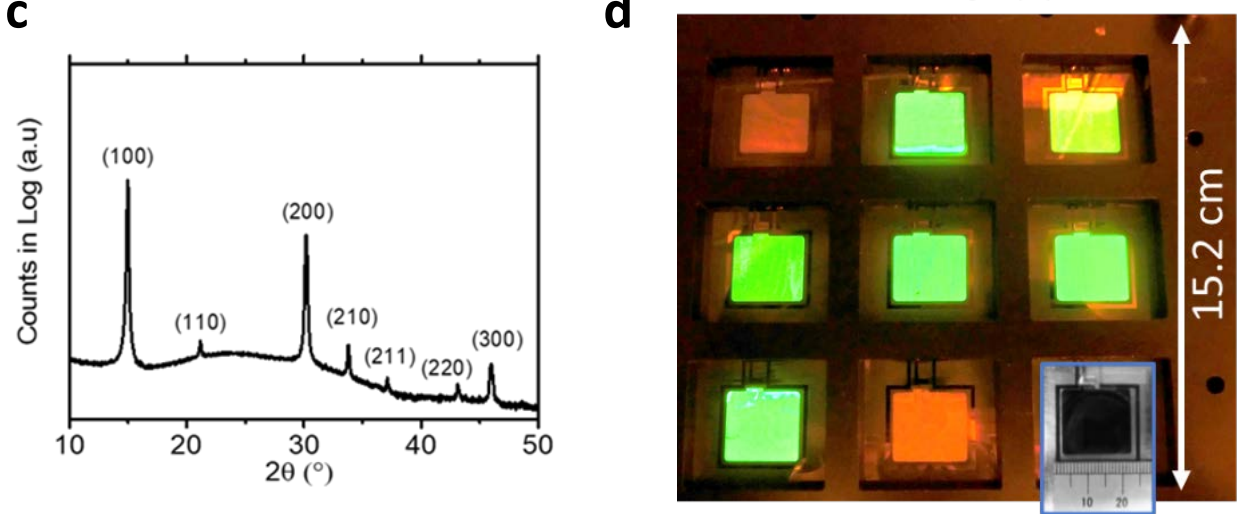

Fig. 1 (a) Schematic illustration of the slot-die coating process combined with the gas-quenching technique. The red and grey arrows indicate the coating and gas quenching directions, respectively. (b) Typical $J-V$ - $L$ curve of a slot-die coated PeLED. Inset: The electroluminescence spectra of a device at $8 \mathrm{~V}$ bias. (c) XRD patterns of the slot-die coated $\mathrm{MAPbBr}_{3}$ film on top of a PEDOT:PSS/Di-NPB bi-layer prepared by gas-quenching. The crystallographic planes are marked on top of the patterns. The broad feature in the baseline is originating from the encapsulation layer. (d) Photograph of the light output from the operational PeLEDs ( 6 devices in ON state) from a slot-die coated substrate, mounted on the $J-V$ - $L$ setup, at $12 \mathrm{~V}$ cumulative bias. The total coated area is $15.2 \times 15.2 \mathrm{~cm}^{2}$. Inset: An image of a single device with an active area of $4.46 \mathrm{~cm}^{2}$ in off state with a scale for size perception.

A typical J-V-L characteristics of the slot-die coated device is shown in Fig. 4b. The PeLED shows a leakage current of $<2 \times 10-3 \mathrm{~mA} / \mathrm{cm} 2$ and a clear diode rectification starting at $4.5 \mathrm{~V}$. The light emission starts at $6 \mathrm{~V}$ and steadily rises to $550 \mathrm{~cd} / \mathrm{m} 2$ before levelling off at $11.5 \mathrm{~V}$. The lower luminance values and higher turn-on voltages of slot-die coated (large area) devices compared to their spin-coated (small area) counterparts can be rationalized in terms of perovskite crystallite size and film thicknesses. Larger perovskite crystallite size $(68 \mathrm{~nm})$ and higher film thickness (more than factor 2) lead to a reduction in radiative recombination3,20 and optical losses37, respectively, in the slot-die coated films. Perhaps more importantly, we point out that the working PeLEDs show rather similar device performance with an average maximum luminance and current efficiencies of $500 \mathrm{~cd} / \mathrm{m} 2$ and $2.4 \mathrm{~cd} / \mathrm{A}$, respectively. The histogram of device performance for a substrate with seven working devices out of nine is shown in Fig. S8 $(E S I+)$. Two of the devices were not measurable due to problems in contacting the electrodes. The devices show uniform green emission centred at $534 \mathrm{~nm}$ (inset Fig. 4b) over the entire active area of $4.46 \mathrm{~cm} 2$, as shown in Fig. 4d. Strategies to further improve the efficiencies of the slotdie coated PeLEDs should largely focus on: (1) coating ultra-thin perovskite emissive layer by optimizing the coating parameters; (2) enhancing the crystallinity by using larger N2 knife at 
higher inlet gas pressures; (3) expediting the rate of supersaturation by using additives in the precursor.

\section{Conclusions}

Gas quenching has been demonstrated as a viable large area compatible method for the formation of high quality $\mathrm{MAPbBr} 3$ films for perovskite light emitting diodes. Smooth and uniform perovskite films with small crystallites can be achieved by initiating the gas-quenching directly after solution deposition. A high gas pressure is preferred for smoother films and, in conjunction with immediate gas-quenching after film deposition, leads to the best device performance. We demonstrate PeLEDs based on MAPbBr3 with a brightness of up to 6600 $\mathrm{cd} / \mathrm{m} 2$ and a current efficiency of up to $7.0 \mathrm{~cd} / \mathrm{A}$. In addition, we translate this scalable fabrication approach to large-area PeLEDs by using the slot-die coating technique. Slot-die coating in combination with gas-quenching allowed us to coat smooth and uniform MAPbBr3 layer over a $231 \mathrm{~cm} 2$ substrate, resulting in large-area PeLEDs with spatially uniform electroluminescence. These PeLEDs exhibit a peak luminance of $550 \mathrm{~cd} / \mathrm{m} 2$ and a current efficiency of $2.6 \mathrm{~cd} / \mathrm{A}$ over an emissive area of $4.46 \mathrm{~cm} 2$. The presented technique offers a strategy that is compatible with established roll-to-roll fabrication techniques. We believe that this strategy can be used to scale up the fabrication of perovskite light-emitting diodes to large areas, potentially even by roll-to-roll manufacturing.

\section{Conflicts of interest}

There are no conflicts to declare.

\section{Acknowledgements}

We thank Mr. Jack van Glabbeek for the help with the thermal evaporator and large area sample preparation. This work was financially supported by the European Commission through the Horizon 2020 Marie Sklodowska-Curie ITN-INFORM project (Grant Agreement 675867).

\section{Notes and references}

1 L. C. Schmidt, A. Pertegás, S. González-Carrero, O. Malinkiewicz, S. Agouram, G. Mínguez Espallargas, H. J. Bolink, R. E. Galian and J. Pérez-Prieto, J. Am. Chem. Soc., 2014, 136, 850-853.

2 Z.-K. Tan, R. S. Moghaddam, M. L. Lai, P. Docampo, R. Higler, F. Deschler, M. Price, A. Sadhanala, L. M. Pazos, D. Credgington, F. Hanusch, T. Bein, H. J. Snaith and R. H. Friend, Nat. Nanotechnol., 2014, 9, 687-692.

3 H. Cho, S.-H. Jeong, M.-H. Park, Y.-H. Kim, C. Wolf, C.-L. Lee, J. H. Heo, A. Sadhanala, N. Myoung, S. Yoo, S. H. Im, R. H. Friend and T.-W. Lee, Science, 2015, 350, 1222-1225.

4 Z. Xiao, R. A. Kerner, L. Zhao, N. L. Tran, K. M. Lee, T.-W. Koh, G. D. Scholes and B. P. Rand, Nat. Photonics, 2017, 11, 108-115.

5 L. Zhang, X. Yang, Q. Jiang, P. Wang, Z. Yin, X. Zhang, H. Tan, Y. (Michael) Yang, M. Wei, B. R. Sutherland, E. H. Sargent and J. You, Nat. Commun., 2017, 8, 15640.

6 J. Li, S. G. R. Bade, X. Shan and Z. Yu, Adv. Mater., 2015, 27, 5196-5202. 
7 M. Yuan, L. N. Quan, R. Comin, G. Walters, R. Sabatini, O. Voznyy, S. Hoogland, Y. Zhao, E. M. Beauregard, P. Kanjanaboos, Z. Lu, D. H. Kim and E. H. Sargent, Nat. Nanotechnol., 2016, 11, 127.

8 L. N. Quan, F. P. García de Arquer, R. P. Sabatini and E. H. Sargent, Adv. Mater., 2018, 30, 1801996.

9 X. Yu Chin, A. Perumal, A. Bruno, N. Yantara, S. A. Veldhuis, L. Martínez-Sarti, B. Chandran, V. Chirvony, A. Shu-Zee Lo, J. So, C. Soci, M. Grätzel, H. J. Bolink, N. Mathews and ad G. Subodh Mhaisalkar, Energy Environ. Sci, 2018, 11, 1770-1778.

10 M. Ban, Y. Zou, J. P. H. Rivett, Y. Yang, T. H. Thomas, Y. Tan, T. Song, X. Gao, D. Credington, F. Deschler, H. Sirringhaus and B. Sun, Nat. Commun., 2018, 9, 3892.

11 Y. H. Kim, H. Cho, J. H. Heo, T. S. Kim, N. S. Myoung, C. L. Lee, S. H. Im and T. W. Lee, Adv. Mater., 2015, 27, 1248-1254.

12 G. Xing, N. Mathews, S. S. Lim, N. Yantara, X. Liu, D. Sabba, M. Grätzel, S. Mhaisalkar and T. C. Sum, Nat. Mater., 2014, 13, 476-480.

13 E. M. Hutter, G. E. Eperon, S. D. Stranks and T. J. Savenije, J. Phys. Chem. Lett., 2015, 6, 30823090.

14 S. D. Stranks and H. J. Snaith, Nat. Nanotechnol., 2015, 10, 391-402.

15 K. Qasim, B. Wang, Y. Zhang, P. Li, Y. Wang, S. Li, S. T. Lee, L. S. Liao, W. Lei and Q. Bao, Adv. Funct. Mater., 2017, 27, 1-9.

16 J. Xing, Y. Zhao, M. Askerka, L. Na Quan, X. Gong, W. Zhao, J. Zhao, H. Tan, G. Long, L. Gao, Z. Yang, O. Voznyy, J. Tang, Z.-H. Lu, Q. Xiong and E. H. Sargent, Nat. Commun., 2018, 9, 3541.

17 J. Wang, N. Wang, Y. Jin, J. Si, Z. K. Tan, H. Du, L. Cheng, X. Dai, S. Bai, H. He, Z. Ye, M. L. Lai, R. H. Friend and W. Huang, Adv. Mater., 2015, 27, 2311-2316.

18 L. Meng, E. P. Yao, Z. Hong, H. Chen, P. Sun, Z. Yang, G. Li and Y. Yang, Adv. Mater., 2017, 29, $1-7$.

19 Z. Wang, T. Cheng, F. Wang, S. Dai and Z. Tan, Small, 2016, 32, 4412-4420.

20 V. Prakasam, F. Di Giacomo, R. Abbel, D. Tordera, M. Sessolo, G. H. Gelinck and H. J. Bolink, ACS Appl. Mater. Interfaces, 2018, 10, 41586-41591.

21 J. Byun, H. Cho, C. Wolf, M. Jang, A. Sadhanala, R. H. Friend, H. Yang and T. W. Lee, Adv. Mater., 2016, 34, 7515-7520.

22 L. Zhao, Y.-W. Yeh, N. L. Tran, F. Wu, Z. Xiao, R. A. Kerner, Y. L. Lin, G. D. Scholes, N. Yao and B. P. Rand, ACS Nano, 2017, 11, 3957-3964.

23 K. Lin, J. Xing, L. N. Quan, F. P. G. de Arquer, X. Gong, J. Lu, L. Xie, W. Zhao, D. Zhang, C. Yan, W. Li, X. Liu, Y. Lu, J. Kirman, E. H. Sargent, Q. Xiong and Z. Wei, Nature, 2018, 562, 245-248.

24 Y. Galagan, F. Di Giacomo, H. Gorter, G. Kirchner, I. de Vries, R. Andriessen and P. Groen, Adv. Energy Mater., 2018, 8, 1801935. 
25 F. Di Giacomo, Y. Galagan, S. Shanmugam, H. Gorter, F. van den Bruele, G. Kirchner, I. de Vries, H. Fledderus, H. Lifka, S. Veenstra, T. Aernouts, P. Groen and R. Andrissen, Proc. SPIE 10363, Organic, Hybrid, and Perovskite Photovoltaics XVIII, 2017, 10363, 103630E.

26 M. Xiao, F. Huang, W. Huang, Y. Dkhissi, Y. Zhu, J. Etheridge, A. Gray-Weale, U. Bach, Y.-B. Cheng and L. Spiccia, Angew. Chem. Int. Ed. Engl., 2014, 53, 9898-9903.

27 F. Huang, Y. Dkhissi, W. Huang, M. Xiao, I. Benesperi, S. Rubanov, Y. Zhu, X. Lin, L. Jiang, Y. Zhou, A. Gray-Weale, J. Etheridge, C. R. McNeill, R. A. Caruso, U. Bach, L. Spiccia and Y. B. Cheng, Nano Energy, 2014, 10, 10-18.

28 B. Conings, A. Babayigit, M. T. Klug, S. Bai, N. Gauquelin, N. Sakai, J. T. W. Wang, J. Verbeeck, H. G. Boyen and H. J. Snaith, Adv. Mater., 2016, 28, 10701-10709.

29 K. Hwang, Y. S. Jung, Y. J. Heo, F. H. Scholes, S. E. Watkins, J. Subbiah, D. J. Jones, D. Y. Kim and D. Vak, Adv. Mater., 2015, 27, 1241-1247.

30 J. Kim, J. S. Yun, X. Wen, A. M. Soufiani, C. F. J. Lau, B. Wilkinson, J. Seidel, M. A. Green, S. Huang and A. W. Y. Ho-Baillie, J. Phys. Chem. C, 2016, 120, 11262-11267.

31 V.-C. Nguyen, H. Katsuki, F. Sasaki and H. Yanagi, Jpn. J. Appl. Phys., 2018, 57, 04FL10.

32 A. Sandström and L. Edman, Energy Technol., 2015, 3, 329-339.

33 R. Abbel, I. De Vries, A. Langen, G. Kirchner, H. T’Mannetje, H. Gorter, J. Wilson and P. Groen, J. Mater. Res., 2017, 32, 2219-2229.

34 K. J. Choi, J. Y. Lee, J. Park and Y. S. Seo, Org. Electron. physics, Mater. Appl., 2015, 26, 66-74.

35 L. Derue, S. Olivier, D. Tondelier, T. Maindron, B. Geffroy and E. Ishow, ACS Appl. Mater. Interfaces, 2016, 8, 16207-16217.

36 F. Di Giacomo, S. Shanmugam, H. Fledderus, B. J. Bruijnaers, W. J. H. Verhees, M. S. Dorenkamper, S. C. Veenstra, W. Qiu, R. Gehlhaar, T. Merckx, T. Aernouts, R. Andriessen and Y. Galagan, Sol. Energy Mater. Sol. Cells, 2018, 181, 53-59.

37 L. Zhao, K. M. Lee, K. Roh, S. U. Z. Khan and B. P. Rand, Adv. Mater., 2018, 1805836, 1805836. 